\title{
Preparation and swelling of hydrophilic magnetic microgels
}

Christine Ménager*, Olivier Sandre, Jérôme Mangili, Valérie Cabuil.

Laboratoire des Liquides Ioniques et Interfaces Chargées, Equipe Colloïdes Inorganiques UMR 7612 CNRS / Université Pierre et Marie Curie (Paris 6)

4 place Jussieu, case 63, 75252 Paris Cedex 05 FRANCE

*to whom correspondence should be addressed : E-mail: menager@ccr.jussieu.fr, Fax: 331 44273675

Keywords : magnetic fluid, microgel, hybrid particles

Abstract : Hydrophilic magnetic microgels were synthesized by a free-radical polymerization in inverse emulsion. Two sizes of magnetic microgels were obtained depending on the initial droplet size of the emulsion. As the polymer matrix is a hydrogel, the novelty of this paper is that the magnetic particles are incorporated from an aqueous magnetic fluid instead of an organic one. In this paper, we underline the physicochemical aspects necessary to control the proper dispersion of the magnetic particles in the polymer matrix. The size, the magnetic content and the swelling degree of micron-sized and sub-micron sized magnetic microgels are characterized. 


\section{Introduction}

The synthesis of hybrid materials by encapsulation of an inorganic material or a liquid in a polymer matrix opens new possibilities in the fabrication of solid particles for materials science. Such composite particles offer potential advantages in applications such as cosmetics, inks and paints coming from the improved compatibility between the filler and the binder [1]. Polymer shells encapsulating inorganic particles are also of great interest in pharmaceutical and biotechnological industries for producing drug release products [2]. However, recent studies have underlined the major difficulty to encapsulate inorganic particles homogeneously, especially in the case of magnetic particles in polymer microgels $[3,4]$.

In the field of drug delivery, many types of colloidal carriers have been tried out, which actively respond to external stimuli such as temperature, $\mathrm{pH}$, electric or magnetic field [5-7]. Among the good candidates, one finds microgels that are cross-linked latex particles swollen by a good solvent.

Aqueous magnetic fluids are colloidal dispersions of magnetic nanoparticles in water, exhibiting a giant paramagnetic behavior. They can be used in biosciences, namely as contrast agents for magnetic resonance imaging (MRI) [8], for magnetic guidance of drugs or radioisotopes and for cell sorting processes.

The encapsulation of magnetic particles inside organic swellable particles made of polystyrene or polymethylmethacrylate has been extensively described [9-10]. In this paper, we describe a general method to encapsulate magnetic particles homogeneously inside a hydrophilic gel or hydrogel. The usual way to produce magnetic polymer particles consists in coating the magnetic material by a surfactant and embedding it in the polymer using processes such as suspension, emulsion or precipitation polymerization. Here we use the inverse emulsion process to disperse the aqueous magnetic fluid and the hydrophilic monomer 
localized together in water droplets just prior to polymerization. As the magnetic particles are themselves hydrophilic, no surface treatment is required before the encapsulation process. This is a key factor since there is neither surfactant nor polymer molecules in the initial magnetic fluid that can interfere with the surfactant used for the emulsification or with the polymer matrix.

\section{Marerials and methods}

\subsection{Materials}

Acrylamide 99\% (AM) was purchased from Aldrich, N,N'-methylenebisacrylamide (MBA) was obtained from Sigma, ferric chloride $\left(\mathrm{FeCl}_{3} 27 \%\right.$ solution), ferrous chloride $\left(\mathrm{FeCl}_{2}\right.$, $\left.4 \mathrm{H}_{2} \mathrm{O}\right)$, ammonia $\left(\mathrm{NH}_{3} 20 \%\right)$, nitric acid $\left(\mathrm{HNO}_{3} 52.5 \%\right)$, hydrochloric acid $(\mathrm{HCl} 35 \%)$, trisodium citrate $\left(\mathrm{C}_{6} \mathrm{H}_{5} \mathrm{Na}_{3} \mathrm{O}_{7}, 2 \mathrm{H}_{2} \mathrm{O}\right)$ and sorbitan monooleate (Span 80) were purchased from Prolabo. V-50 (2-2' azobisamidino propane) was obtained from Fluka.

\subsection{Synthesis of the aqueous magnetic fluid:}

$180 \mathrm{~g}$ of $\mathrm{Fe}^{\mathrm{II}}$ salt $(0.9$ mole $)$ and $715 \mathrm{~mL}$ of $\mathrm{Fe}^{\mathrm{III}}$ solution $(1.5$ moles $)$ were mixed in acidic medium $(\mathrm{HCl})$. Coprecipitation of magnetite $\left(\mathrm{Fe}_{3} \mathrm{O}_{4}\right)$ particles was performed by rapid addition of concentrated ammonia (1L). After acidification with a nitric acid solution (360 $\mathrm{mL}$ ), the addition of $800 \mathrm{~mL}$ of a boiling solution of ferric nitrate ( $323 \mathrm{~g}, 1.3$ moles) leaded to the chemical oxidation of magnetite to maghemite $\left(\gamma \mathrm{Fe}_{2} \mathrm{O}_{3}\right)$. After magnetic decantation, the red precipitate was dispersed in water and then $70 \mathrm{~g}$ of trisodium citrate salt $(0.24$ mole $)$ was added. The mixture was heated at $80^{\circ} \mathrm{C}$ for $1 / 2 \mathrm{~h}$. The magnetic material was precipitated by addition of acetone. The precipitate was isolated and suspended in distilled water leading to a stable colloidal dispersion $(\mathrm{pH} 7)$.

\subsection{Emulsion polymerization}

Two series of micron-sized and sub-micron sized microgels were synthesized, both of them being based on polyacrylamide cross-linked by N,N'-methylenebisacrylamide (MBA). 
Both microgels were synthesized using an inverse (W/O) emulsion polymerization method. Aqueous droplets were stabilized by an emulsifier (Span 80) and dissolved in dodecane. In a first step, the polar monomer and the cross-linker were solubilized directly in the magnetic fluid to be incorporated in the final microgels. In a second step, this aqueous mixture was introduced under stirring into the oily phase. Polymerization was started by the introduction of a free radical initiator (V50). Using either inverse emulsions or miniemulsions, it is possible to investigate two ranges of sizes : the micron or the sub-micron one respectively.

2.3.1 Polymerization in emulsion: We investigate here two weight ratios MBA/AM of crosslinker with respect to the monomer (5 wt $\%$ and $2.5 \mathrm{wt} \%)$ at a constant monomer concentration (28 wt\% AM in the aqueous magnetic phase). The hydrophilic monomer acrylamide (AM, 2g) and the cross-linker N,N'-methylenebisacrylamide (MBA, 0.1g and $0.05 \mathrm{~g}$ respectively for the two investigated weight fractions were solubilized in $5 \mathrm{~mL}$ of the pH7 magnetic fluid. Both aqueous phase and organic solution of the emulsifier (Span 80 in $15 \mathrm{~mL}$ dodecane, $m_{\mathrm{Span} 80} / m_{\text {dod }}=2.25 \%$ ) were placed under $\mathrm{N}_{2}$ for $1 / 2 \mathrm{~h}$ and then added together and stirred. Polymerization was initiated by adding $20 \mathrm{mg}$ of $\mathrm{V}-50$ solubilized in $0.5 \mathrm{~mL}$ of water $(1 \mathrm{wt} \% / \mathrm{AM})$ and raising temperature to $50^{\circ} \mathrm{C}$ in a water bath. The reaction mixture was stirred one hour at $50^{\circ} \mathrm{C}$ under $\mathrm{N}_{2}$. At the end of the reaction, the mixture had become heterogeneous: the magnetic microgels were settling at the bottom of the flask, the biggest ones being often stuck together. However, the magnetic microgels were easily separated from the organic solvent under a magnetic field gradient (a strong Na-Fe-B magnet) and washed several times with ethanol. They were then redispersed in water but due to their size, such suspensions of magnetic microgels in water were not stable.

2.3.2 Polymerization in miniemulsion : the magnetic miniemulsions were prepared under the same conditions as the emulsions except for mixing, which differs by the use of ultra-sons. After degassing, the organic phase (0.3 g Span 80 and $11 \mathrm{~g}$ dodecane) was mixed with the 
aqueous solution (1.4 g AM, $0.07 \mathrm{~g} \mathrm{MBA}$ in $1.9 \mathrm{~mL}$ of the aqueous magnetic fluid). The radical initiator $(20 \mathrm{mg}$ V50 in $0.5 \mathrm{~mL}$ of water) was introduced at the end (after nitrogen bubbling). After vortex stirring (5 minutes) the mixture was placed in an ultrasonic bath (Elma Transsonic T 460H, 285 Watts, $35 \mathrm{kHz}$ ). The reaction mixture was sonicated at $50^{\circ} \mathrm{C}$ for 15 minutes. After reaction, we always noted the presence of a coagulum and a supernatant. The microgels in the supernatant were characterized in their solvent of synthesis and also after several washings in ethanol and finally in water.

\subsection{Determination of the magnetic load}

The mass fraction of magnetic material inside the composite microgels was obtained from the ratio of the iron oxide weight divided by the total dry weight of a given sample. In the case of the stable sub-micron microgels, magnetic measurements could be performed with a vibrating sample magnetometer because the suspension did not settle down under the strong applied magnetic field (up to 1 tesla).

Concerning the larger micron-sized microgels, the molar content of iron was titrated by atomic absorption spectroscopy with a Pelkin Elmer Analyst 100 apparatus after degradation of the particles in boiling $\mathrm{HCl}(6 \mathrm{~mol} / \mathrm{L})$.

\subsection{Optical microscopy}

The samples were observed with an inverted microscope (Leica DMIL, 40x magnification). The orange color of the microgels is due to the trapped magnetic nanoparticles that strongly absorb in the visible spectrum. The pictures recorded with a color video camera are digitized with a PC graphic card (ATI All-In-Wonder Pro).

\subsection{Transmission Electron Microscopy (TEM)}

TEM was performed using a JEOL 100CXII top entry UHR electron microscope. The diluted solutions were deposited on a carbon-coated copper grid and left to dry in the air. No further 
contrasting technique was applied. In the case of miniemulsions only, the microgels were deposited in their solvent and then washed directly on the grid with ethanol.

\subsection{Multi-angle Photon Correlation Spectroscopy (PCS)}

PCS was performed on a Macrotron spectrometer (Amtec) equipped with a $\mathrm{He} / \mathrm{Ne}$ laser $(\lambda=632.8 \mathrm{~nm})$ and a Malvern 7132 correlator card with 192 time channels. Samples (about 1 $\mathrm{mL}$ in cylindrical glass vials) were immersed in a filtered toluene bath, which temperature was regulated $\left( \pm 0.2^{\circ} \mathrm{C}\right)$ by a water jacket. The autocorrelation functions of the scattered intensity were recorded using a $0.5 \mu$ s sampling time and 30 sub-runs of $20 \mathrm{~s}$ averaged together for a total duration of $10 \mathrm{~min}$. The scattering angle $\theta$ was varied from $45^{\circ}$ up to $155^{\circ}$ every $5^{\circ}$. Precise measurements of the hydrodynamic diameter by PCS necessitate the exact knowledge of the solvent viscosity $\left(\eta^{\text {solvent }}\right)$ and of the refraction $\left(n^{\text {solvent }}\right)$. Therefore PCS could not be performed directly in the synthesis emulsion which contains many species (polymer, surfactant, ...) which modify those two parameters. Indeed the particles were isolated with a strong permanent magnet, washed several times and resuspended in filtered absolute ethanol $\left(\eta^{\mathrm{EtOH}}=1.159 \mathrm{cPoise}\right.$ at $22^{\circ} \mathrm{C}$ and $\left.n^{\mathrm{EtOH}}=1.3611\right)$ or freshly distilled water $\left(\eta^{\text {water }}=0.957 \mathrm{cPoise}\right.$ at $22^{\circ} \mathrm{C}$ and $\left.n^{\text {water }}=1.3326\right)$. Those values of dynamic viscosities were calculated from the solvent densities $\left(\rho^{\text {water }}=1, \rho^{\mathrm{EtOH}}=0.7937\right)$ and experimental values of the kinematic viscosities $\left(v^{\text {water }}=0.957 \mathrm{cStokes}, v^{\mathrm{EtOH}}=1.461 \mathrm{cStokes}\right.$ at $\left.22^{\circ} \mathrm{C}\right)$ measured with an Ubbelohde viscosimeter (capillary No. I, Schott-Geräte, Germany). Tabulated values were used for the indexes of refraction. The autocorrelation functions were analyzed using the standard program CONTIN [11-12], in order to extract the dominant decay time $\tau^{\text {max }}$. Then the decay rate $\Gamma^{\max }=\left(\tau^{\max }\right)^{-1}$ was used in the primary equation of PCS theory:

$$
\Gamma^{\max }=\frac{k_{\mathrm{B}} T}{3 \pi d_{H}} \frac{q^{2}}{\eta^{\text {solvent }}}
$$


where the scattering vector writes: $\quad q=\frac{4 \pi n^{\text {solvent }}}{\lambda} \sin \left(\frac{\theta}{2}\right)$

The hydrodynamic diameters $d_{\mathrm{H}}$ were computed from the slope of a linear regression of $\Gamma^{\mathrm{max}}$ as a function of $q^{2} / \eta^{\text {solvent }}$ extrapolated in the zero $\mathrm{q}$ limit. The reliability of this method was tested on monodisperse beads of (Stöber) silica with $d_{\mathrm{TEM}}=278 \mathrm{~nm}$, for which the linear regression yields $d_{\mathrm{H}}=288 \mathrm{~nm}$ (the discrepancy of $10 \mathrm{~nm}$ can be attributed to a hydration layer).

Analyzing the auto-correlation functions by the method of cumulants rather with the CONTIN program decreases the quality of the linear regressions of $\Gamma^{\max }$ vs. $q^{2} / \eta^{\text {solvent }}$, presumably due to the influence of a secondary characteristic time (see inset in Figure 5a). This mode approximately ten times faster than the main one corresponds to a hydrodynamic diameter about $15 \mathrm{~nm}$, therefore attributed to Brownian diffusion of traces of magnetic nanoparticles released from the gel into the solution.

This mode is ten times faster than the main one and therefore attributed to Brownian diffusion of traces of magnetic nanoparticles released from the gel into the solution.

\section{Results and discussion}

All related studies revealed that a successful encapsulation first requires a good dispersion of the inorganic particles in the medium prior to carrying out the polymerization [13]. In fact, this is a prerequisite to their use in a variety of applications. The magnetic load must be uniformly dispersed in the gel. The clustering of the magnetic nanoparticles in a localized spot inside the composite colloids has often been observed in the literature [3].

3.1 Compatibility between the magnetic fluid and the polymer

The encapsulated magnetic nanoparticles are maghemite $\left(\gamma \mathrm{Fe}_{2} \mathrm{O}_{3}\right)$ grains, which were synthesized using a method described above and elsewhere [14]. The diameters of particle 
range between 5 and $14 \mathrm{~nm}$. This rather broad size distribution can be narrowed through a post synthesis treatment $[15,16]$. In order to avoid aggregation of the ferric oxide particles in the polymer network, we used a magnetic fluid that is stabilized in water at $\mathrm{pH}$. The trapping of the same kind of particles in a polymer network has already been described in the case of bulk (centimeter large) hydrogels $[17,18]$. These magnetic nanoparticles are covered by citrate ligands and are negatively charged at $\mathrm{pH}$. The electrostatic repulsions ensure the stability of the colloidal dispersion. The control of the stability of the inorganic particles dispersions during emulsification and polymerization appears to be the major issue [19]. The volume fraction of magnetic particles set to $2.3 \%$ and a concentration of unadsorbed citrate species in the aqueous phase equal to $2.510^{-2} \mathrm{~mol}^{-1}$ are appropriate to avoid any microscopic destabilization of the colloidal dispersion after mixing with the monomer. The volume fraction of magnetic particles was determined by magnetic measurements. The magnetization curve was measured with a vibrating sample magnetometer [20]. A fit of this curve allows the determination of the volume fraction of maghemite nanoparticles $(\phi=2.3 \%$ from the magnetization at saturation and of the size distribution modeled by a Log-normal law $\left(\mathrm{d}_{0}=7 \mathrm{~nm}, \sigma=0.4\right)$.

Depending on technical applications, it is also necessary to control the size of the microgels. For this purpose, two kinds of synthesis are described here, leading to final microgels either in the micrometer or in the sub-micrometer range of size.

\subsection{Micron-sized microgels}

\subsubsection{Magnetic behavior and magnetic content}

Due to their size, the microgels prepared in emulsions and dispersed in water can be observed using optical microscopy (Figure 1a). The microgels are spherical just like the emulsion droplets and their diameters vary between 10 and $60 \mu \mathrm{m}$. When a magnetic field of low intensity (200 Gauss) was applied, we observed the chaining of the magnetic microgels with 
perfect reversibility (Figure 1b). Please note that there was no visible deformation of the sphere (magneto-striction) at least for this low field intensity.

The determination of the magnetic load inside those microgels yielded $64 \mathrm{wt} \%$ for the larger cross-linking density (5\%wt MBA/AM) and $34 \mathrm{wt} \%$ for the lower one (2.5\%wt MBA/AM).

\subsubsection{Swelling degree and dynamics}

Microgels swell in suitable solvents and their swelling degree depends on the concentration of cross-linker. We followed the swelling of microgels (5 and $2.5 \mathrm{wt} \% \mathrm{MBA} / \mathrm{AM}$ ) individually under the microscope. The microgels were deposited from an alcoholic suspension onto a glass slide; after evaporation of ethanol, the beads of collapsed gel were allowed to hydrate by adding a water droplet on them. The swelling process was recorded and shown on Figure 2.

Thanks to the coloration by iron oxide, we observe the flow of solvent towards the center of the microgel concomitant with the increase of its external diameter. The flow of solvent (water) inside the microgel is concomitant with the increase of its diameter, as seen on the plot of the diffusion front (closed markers) and of the external diameter (open markers) as a function of time (Figure 2). The degree of cross-linking affects the swelling ratio significantly: qualitatively a $2.5 \mathrm{wt} \%$ cross-linked microgel absorbs about twice the volume of water of a $5 \mathrm{wt} \%$ MBA microgel for the same total monomer concentration.

These experiments to follow kinetics of minigel swelling are reminiscent of those reported for polyacrylamide spherical macrogels with diameters of the order of the millimeter [21]. Interestingly, the characteristic time of swelling $\tau$ is about $10^{4}-10^{5}$ shorter in the case of minigels, in accordance with the theory of Tanaka et al. that shows that $\tau$ scales with the square of the bead diameter. On these pictures, we also observe the transient rippling instability of hydrogel surface, which was already reported for centimeter large hydrogels with a surface constrained to adhere on a substrate and a free interface with the swelling solvent [22]. 
Please note that there is no detectable release of the magnetic content, this reversible swelling resulting only from the diffusion of water and ions from the outer solution towards the porous medium constituting the hydrogel.

\subsubsection{TEM analysis}

Due to their inorganic load, the magnetic microgels can be analyzed by TEM without any coloration, the electronic contrast being already provided by the magnetic particles (Figure 3a). The magnetic particles are well dispersed in the gel, the darker color at the center compared to periphery being due to a larger thickness. Figure $3 \mathrm{~b}$ shows a magnification of the microgel sphere at the periphery. Although the density of the film is high, we can still visualize individual particles, which means that they are not distributed as islands. In addition, the saw-tooth profile of the gel wedge is attributed to a wetting instability particular to elastic microgels that will be developed in a forth-coming paper.

\subsection{Sub-micron sized microgels}

Ugelstad et al [23] first published results on miniemulsions polymerization leading to polystyrene polymer particles. The principles of miniemulsion polymerization in non-aqueous systems (inverse miniemulsions) have been described to produce hydrophilic polymer latexes [10]. It has been shown that the addition of salt has a positive effect on the stability of the inverse miniemulsion by acting as osmotic pressure agent preventing Ostwald ripening. Here the salt was added with the magnetic fluid (tri-sodium citrate salt).

\subsubsection{Magnetic content}

The magnetic content has been calculated from the magnetic measurement made on stable colloidal dispersion of magnetic microgels in water and from the dried mass of polymer. For a sample with an overall solid fraction (dry weight /total weight) of $0.77 \mathrm{wt} \%$, we measured a magnetization at saturation $M_{\mathrm{s}}=330 \mathrm{~A} / \mathrm{m}$ (Figure 4). Using the specific magnetization of colloidal maghemite $m_{\mathrm{s}}=3.110^{5} \mathrm{~A} / \mathrm{m}$ which is experimentally about $75 \%$ of the value for 
bulk maghemite [24], we obtain the volume fraction of magnetic particles $\phi=M_{\mathrm{s}} / m_{\mathrm{s}}=0.106 \%$ and thus their mass fraction $0.53 \mathrm{wt} \%\left(\rho=5 \mathrm{~g} \cdot \mathrm{cm}^{-3}\right)$. The magnetic mass fraction is thus 0.53 $/ 0.77=69 \mathrm{wt} \%$ in these microgels.

\subsubsection{Swelling degree}

Contrary to samples polymerized in conventional emulsions, the miniemulsions were stable (remained dispersed) several weeks after polymerization in their solvent (dodecane). We did not check all the criterions to ensure that the presented system could really be qualified as a polymerization in miniemulsion, but it corresponds to miniemulsion in terms of stability and of particles size. Polymerization in miniemulsions with $5 \mathrm{wt} \% \mathrm{MBA} / \mathrm{AM}$ and $42 \mathrm{wt} \% \mathrm{AM}$ monomer in the aqueous magnetic phase allowed to get magnetic microgels that exhibit colloidal stability.

By performing multi-angle photon correlation spectroscopy (PCS) on dispersions of microgels in bad and good solvent respectively, we were able to demonstrate a change in size in response to a change in solvency. By plotting the dominant correlation rate $\Gamma^{\max }$ versus the square of the scattering vector divided by the solvent viscosity, $q^{2} / \eta^{\text {solvent }}$, we obtain the hydrodynamic diameter from the inverse of the slope near the origin: $d_{\mathrm{H}}{ }^{\mathrm{EtOH}}=158 \mathrm{~nm}$, $d_{\mathrm{H}}{ }^{\text {water }}=195 \mathrm{~nm}$ and $d_{\mathrm{H}}=288 \mathrm{~nm}$ for the test beads (Figure 5a). As a result, multi-angle PCS measurements and extrapolation to the low-regime enabled to bypass the issue of polydispersity, thereby reporting a significant increase $(+23.4 \%)$ of the hydrodynamic diameters (Figure 5a). The microgels thus undergo (on average) a $(1.234)^{3} \sim 1.9$-fold increase in volume when they are transferred from a bad solvent (ethanol) into a good solvent (water) [25]. Please note that this measurement of the hydrodynamic diameter represents the (volume) averaged value among a large distribution of diameters. Unlike the straight line over the whole range of $q$ vectors obtained with monodisperse silica beads, the curves on Figure 4a for minigels both in ethanol and water exhibit a positive deviation from linearity at large $q$. This 
upward curvature is attributed to a large polydispersity of the samples, estimated as a Lognormal distribution with $\sigma=0.45$ from the width at half-height of the CONTIN peak.

\subsubsection{TEM analysis}

Figure $5 \mathrm{~b}$ is a TEM picture of microgels synthesized in dodecane. Most of them appear spherical and the measurement of diameters $\left(d_{\text {TEM }}\right)$ over 82 minigels shows a Log-normal law of peak value $d_{0}=65 \mathrm{~nm}$ and standard deviation $\sigma=0.25$ (no unit).

Interestingly, all the microgels are loaded with magnetic nanoparticles, which appear homogeneously distributed within the polymer.

\section{Conclusion}

Rapid and reversible response to a weak magnetic field, solvent swelling without release of the magnetic content and homogeneous distribution of the magnetic colloid inside the matrix, these are the major properties of the hydrophilic magnetic microgels described here. All these properties concomitant with a high magnetic oxide content are essential for biotechnological applications. Here we have described a simple method of homogeneous encapsulation of inorganic particles in hydrogel microbeads. The monomer was just added to the colloidal solution made of inorganic particles in water avoiding any destabilization of the colloidal solution by the careful adjustment of the ionic strength and the $\mathrm{pH}$. However the homogenization of the miniemulsion could be improved by means of turbidity and surface tension measurements as described by Landfester [9].

The process described in this paper is widely applicable to a large variety of water-soluble polymers (polyNIPAM for instance) and to inorganic materials that can be dispersed as colloidal solutions. Moreover, it allows the co-encapsulation of the magnetic fluid with water soluble drugs or markers for biomedical applications. 


\section{Acknowledgments}

The authors thank Chamsoudine Youssouf for preliminary experiments. The electron microscopy was performed by Fabienne Warmont of the "Service de microscopie électronique de l'Université Paris 6".

\section{References}

[1] Murray MJ, Snowden MJ, Advances in Colloid and Interface Science 1995; 54:73.

[2] Elaissari A, Bosc E, Pichot C, Mandrand B, Bibette J, Mondain-Monval O, French Patent $2800635,1999$.

[3] Landfester K, Adv. Mater. 2001; 13: 765.

[4] Landfester K, Ramirez L.P, J. Phys.: Condens. Matter. 2003; 15: S1345.

[5] Lee KY, Peters MC, Mooney DJ, Adv. Mater. 2001; 13: 837.

[6] Sauer M, Streich D, Meier W, Adv. Mater. 2001; 13: 1649.

[7] Kiser PF, Wilson G, Needham D, Nature 1998; 394: 459.

[8] Billotey C, Wilhelm C, Devaud M, Bacri J-C., Bittoun J, Gazeau F, Magnetic Resonance in Medicine 2003; 49(4): 646.

[9] Hoffmann D, Landfester K, Antonietti M, Magnetohydrodynamics 2001; 37: 217.

[10] Landfester K, Macromol. Rapid. Commun. 2001; 22: 896.

[11] Provencher S, J. Chem. Phys. 1976; 64: 2772.

[12] Provencher S, Comput. Phys. Commun. 1982; 27: 213.

[13] Erdem B, Sudol ED, Dimonie VL, El-Aasser MS, J. Polym. Sci. A Polym. Chem. 2000; 38: 4431.

[14] Massart R, IEEE Trans. Magn. Mater. 1981; 17: 1247

[15] Massart R, Cabuil V, Journal de Chimie Physique. 1987; 84: 967. 
[16] Massart R, Dubois E, Cabuil V, Hasmonay E, J. Magn. Magn. Mat. 1995; 149: 1.

[17] Mayer CR, Cabuil V, Lalot T, Thouvenot R, Adv. Mater. 2000; 12: 417.

[18] Galicia JA, Sandre O, Cousin F, Guemghar D, Ménager C, Cabuil V, J. Phys.: Condens. Matter. 2003; 15:S1379.

[19] Erdem B, Sudol ED, Dimonie VL, El-Aasser MS, J. Polym. Sci. A Polym. Chem. 2000; 38: 4441 .

[20] Foner S, Macniff EJ, The Rev. Scient. Instr. 1968; 39: 171

[21] Tanaka T, Fillmore DJ, J. Chem. Phys. 1979; 70: 1214.

[22] Tanaka T, Sun S-T, Hirokawa Y, Katayama S, Kucera J, Hirose Y, Amiya T, Nature 1987; 325: 796.

[23] Ugelstad J, El-AMsser MS, Vanderhoff JW, Polym. Lett. Ed. 1973; 11:503.

[24] Bacri JC, Perzynski R, Salin D, Cabuil V, Massart R, Journal of magnetism and magnetic materials 1986; 62: 36 .

[25] This measurement of the swelling ratio for sub-micrometric minigels is slightly smaller than expected from the optical pictures of Figure 2 representing larger minigels in the same solvent conditions. In that case the diameter increased up to $+50 \%$. However those minigels stuck to glass were spherical caps rather than complete spheres, so that the volume change was not easily computed from the cubic power of ratio of diameters (one would also need to measure the change of contact angle). 


\section{Figure legends}

Figure 1: Optical micrographs of magnetic microgels (5\%wt MBA/AM) prepared in water in oil emulsion and dispersed in water a) without magnetic field b) under magnetic field ( $\mathrm{B}=200$ Gauss). The scale bar measures $20 \mu \mathrm{m}$.

Figure 2: Optical micrographs of the swelling dynamics of magnetic microgels $(5 \% \mathrm{wt}$ MBA/AM) deposited from an ethanol suspension onto a glass slide. The scale bar measures $20 \mu \mathrm{m}$. The orange color is due to the encapsulation of magnetic nanoparticles. a) Initially in the dried state b) $0.7 \mathrm{~s} \mathrm{c}$ ) $1.8 \mathrm{~s} \mathrm{~d}$ ) $3.9 \mathrm{~s}$ e) $6.6 \mathrm{~s} \mathrm{f}$ ) $16 \mathrm{~s}$ after the addition of water. The flow of solvent (water) inside the microgel is concomitant with the increase of its diameter, as seen on the plot (g) of the diffusion front (closed markers) and of the external diameter (open markers) as a function of time.

Figure 3: a) TEM image of a microgel (5\%wt MBA/AM) prepared in emulsion. The microgel spreads out onto the copper grid coated by a carbon film; the bar length is $1 \mu \mathrm{m}$; b) At higher magnification, a dense film of magnetic particles is visualized; the bar length is $100 \mathrm{~nm}$.

Figure 4: Magnetization curve of sub-micron sized microgels measured with a vibrating sample magnometer [19]. The upward and downward oriented triangles represent the field rise and field decrease respectively. The absence of hysteresis loop indicates a pure superparamagnetic behavior of the minigels. The solid line is a fit by the Langevin law averaged by a log-normal distribution law of the diameters $P(d)$ plotted in the inset $\left(d_{0}=6.9\right.$ $\mathrm{nm} ; \sigma=0.37)$ and by the particles volumes, the dash line representing the volume distribution law $\mathrm{d}^{3} \mathrm{P}(\mathrm{d})$.

Figure 5: a) PCS measurements on magnetic minigels prepared in a water/dodecane miniemulsion redispersed in ethanol (closed circles) and in water (open circles) and standard 
curve obtained for monodisperse silica beads (triangles) of diameter $d_{\mathrm{TEM}}=278 \mathrm{~nm}$. The inset shows an example of decay time's distribution obtained from a CONTIN fit of the autocorrelation function of the intensity scattered at $95^{\circ}$ by a suspension of minigels in ethanol ; b) TEM image of sub-micrometer microgels prepared from miniemulsions $(5 \% \mathrm{wt}$ MBA/AM) in dodecane. The bar length is $100 \mathrm{~nm}$.

Figures

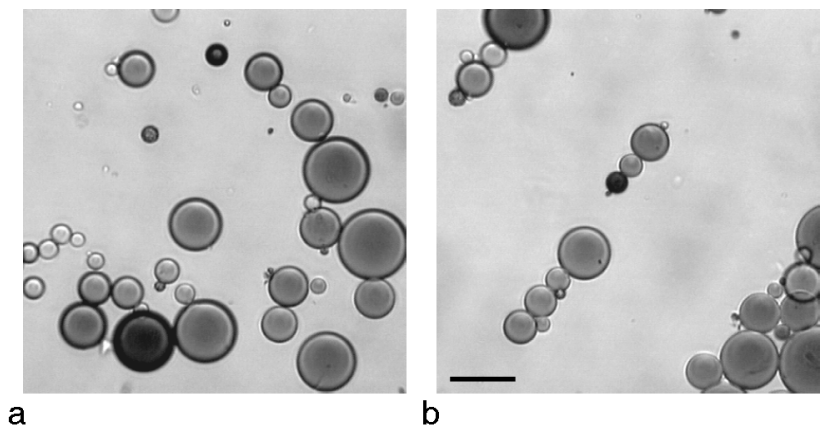

Figure 1 


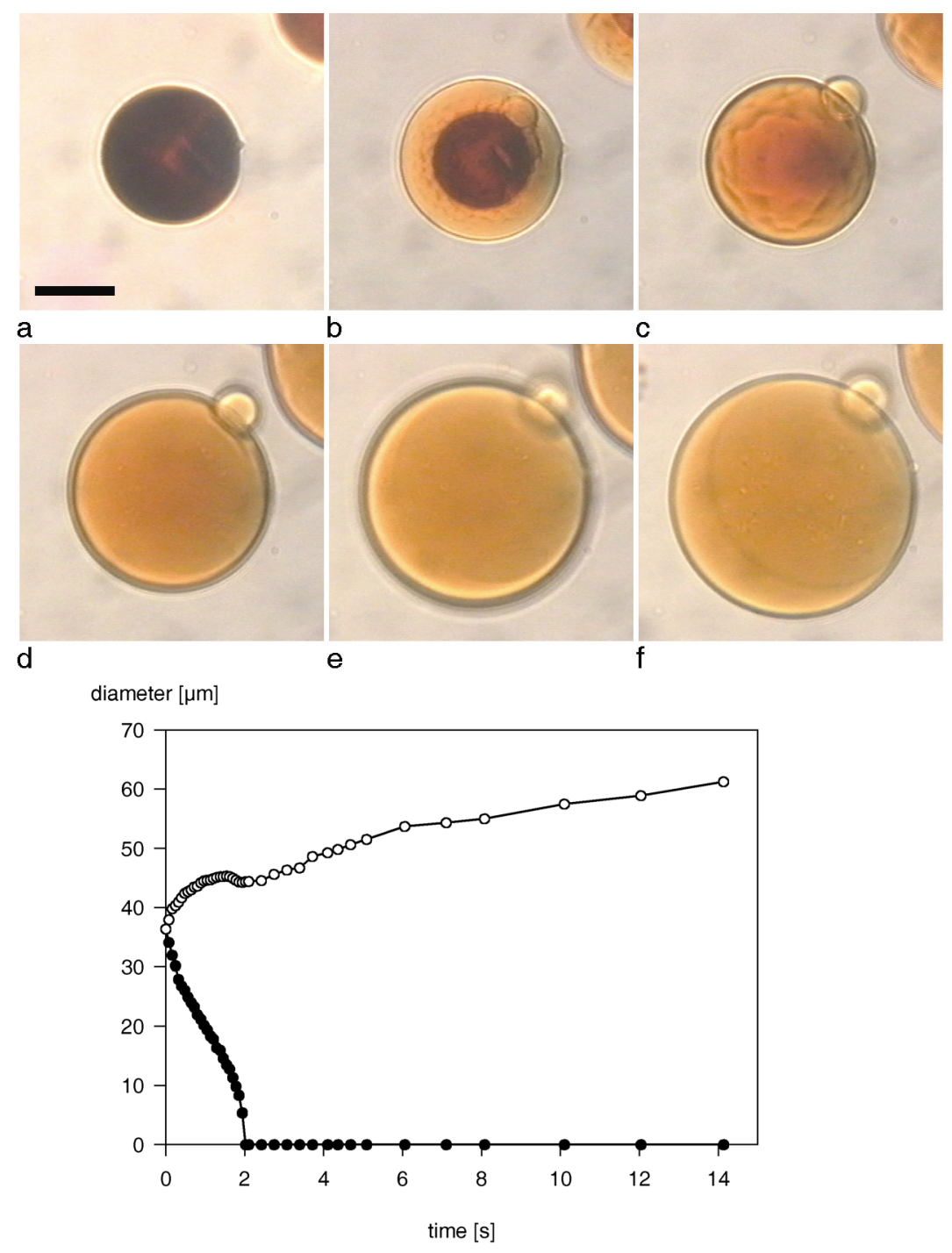

Figure 2

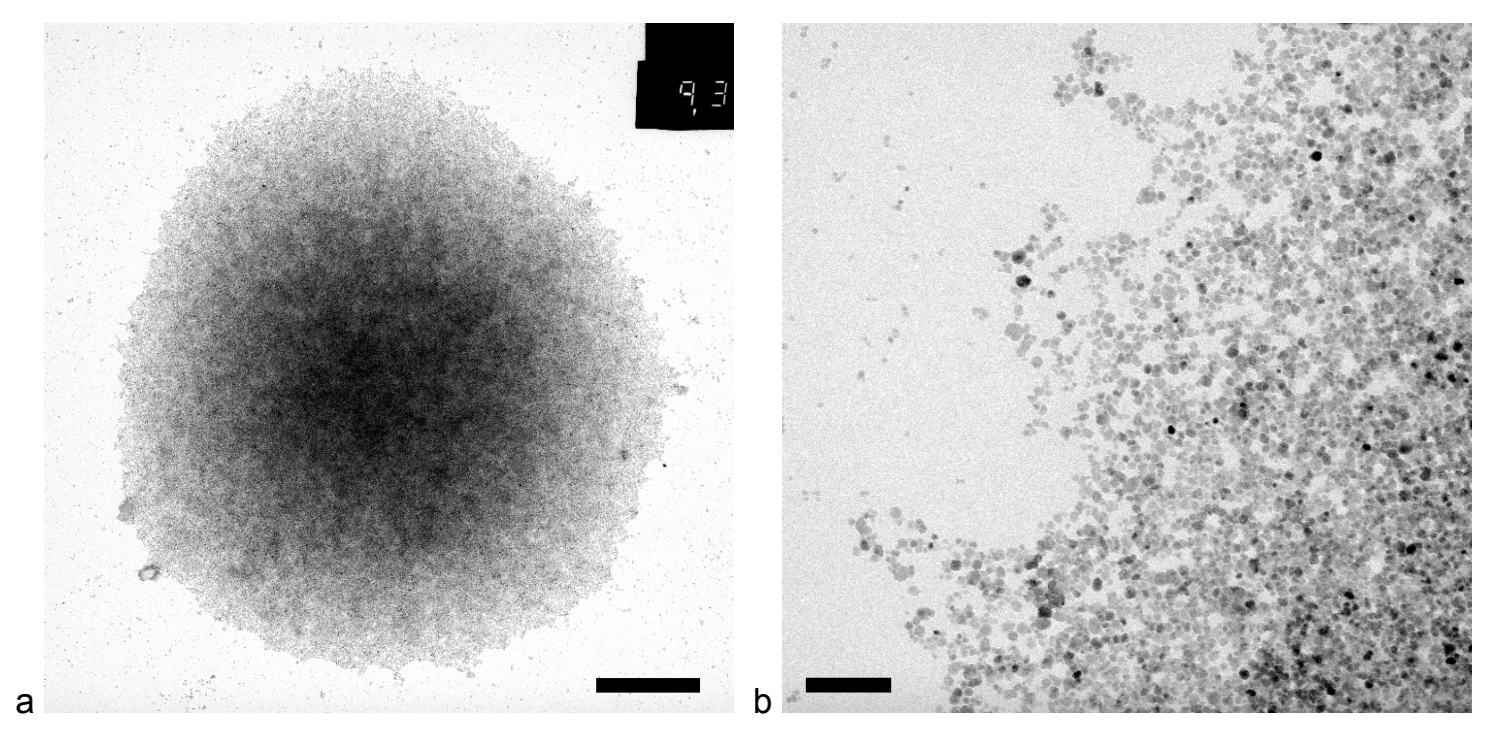


Figure 3

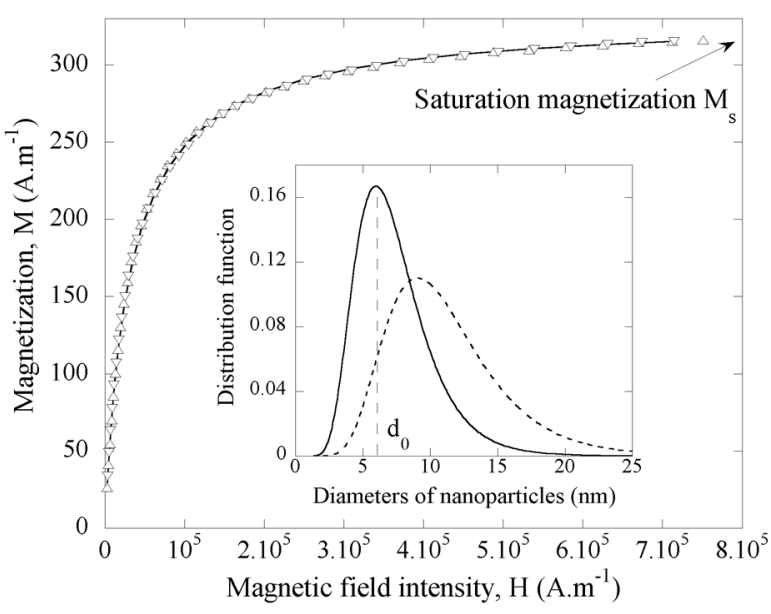

Figure 4
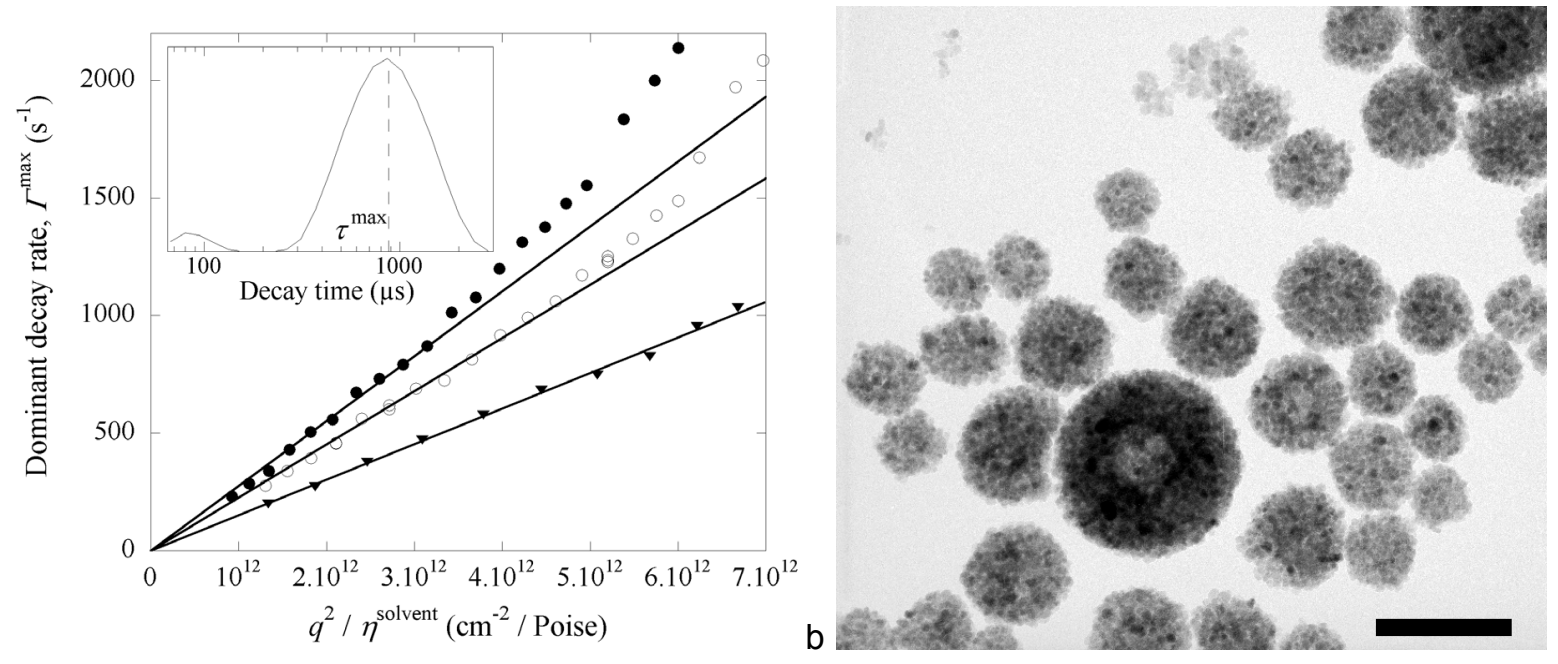

Figure 5 